\section{Are these the insulins of the future?}

J.Markussen's group at Novo published two papers in the previous issue of Protein Engineering on a novel combination of protein engineering by genetic manipulation and chemical semisynthesis. These papers demonstrate that the two methods are, in the given system, much more powerful than either alone.

However, the thoroughness with which the authors document both this conclusion, and their physico-chemical studies on their new analogues, should not cause us to overlook another aspect, namely that these two papers are the first public indication that Novo may have taken a significant step towards a new generation of insulins. These insulins might permit a real improvement in the control of mobilization from the injected subcutaneous depot.

The analogue in which $\mathrm{Thr}^{\mathrm{B} 27}$ is exchanged for an arginine residue, and in which the C-terminus of the B-chain is amidated has, as one would expect, a pI considerably higher than that of the native hormone. What could not have been predicted is the effect of these substitutions on the crystallization properties of the product.

The ability of native insulin to form remarkably uniform rombohedral crystals ( $\sim 25 \mu \mathrm{m}$ along each edge) is well known and has been the basis of many of Novo's long-acting preparations. If one injects a preparation containing a suspension of these crystals as a subcutaneous depot, they take a considerable time to dissolve. Thus, the crystalline insulin is mobilized more slowly from the depot than would be the case for soluble, or amorphous, insulin. It is obvious from the papers under discussion that Novo are now at least considering dispensing the new insulin analogues as a solution (mildly acidic, but unbuffered). The $\mathrm{pH}$ of the injected depot would soon rise, due to the buffering power of the tissue fluids, and during this phase there could be an initial period of rapid mobilization of the soluble analogue. Soon, however, a $\mathrm{pH}$ value would be reached at which the analogue will suddenly crystallize, giving thereafter a much slower insulin release. The tendency to crystallize seems to depend very sharply on zinc content, and it would appear that different preparations could be made having different kinetics of crystallization, and thus of mobilization, depending on the amount of zinc present.

In what way does all of this constitute an advance with a real prospect of benefits to patient care? It is obviously too early to say, and Markussen's group are careful not to make any exaggerated claims. However, if all went well, even the simple fact of being able to dispense insulin as a solution could in itself be a considerable help to the patient, in that it would permit him or her always to inject precisely the same dose. Current crystalline suspensions need to be shaken to homogeneity before injection, and tests show that this is surprisingly difficult to do, with cumulative effects on the actual dose employed as a given bottle is used up. Also, many of the newer forms of injection equipment, much more acceptable to the patient, do not lend themselves to shaking of any kind. (If the reader feels that mundane details of this kind are out of place in a scientific research journal, he or she is unlikely to be a diabetic. The rest of us might wish to recall just how many of our fellow citizens are obliged to build their lives around factors of this sort.)

The biphasic release mentioned above may well prove too difficult to control reliably. It might well prove necessary always to work at concentrations of zinc that, while low, have already more or less suppressed the rapid phase. However, this would still leave an immensely useful range of conditions in which the long-acting phase of mobilization could be subjected to fine tuning with a facility and precision never before possible.

Even from the details given in these papers, it seems clear that such analogues (as well as native human insulin) could be made easily on an industrial scale by the new methods. Only one major problem appears to remain before attempting to see by experiment if this type of analogue could in practice realize its promise for improved treatment. Will they be immunogenic? No data are yet offered, and we must await events. However, the most desirable of the substitutions look to be less likely to confer immunogenicity on the analogue than do, for example, the three natural substitutions that separate bovine from human insulin. Bovine insulin has been one of the mainstays of insulin therapy in Man for more than 50 years.

\section{Robin E. Offord \\ Département de Biochimie Médicale Centre Médical Universitaire \\ 9, avenue de Champel 1211 Genève 4, Suisse}

\section{Commission of the European Communities: Biotechnology Action Programme (BAP) \\ Conference on 'Enzyme engineering: protein design and applications in biocatalysis', Capri, Italy, May 2-6, 1987}

It was in the Certosa San Giacomo gallery, with its medieval quadrangle that, during five days in May, twentieth century European biotechnology assessed its progress against a backdrop of seventeenth century Italian art. The BAP programme, as it is now called, was established in 1985 as a successor to the pioneering Biomolecular Engineering Programme (BEP) (1982-1986) which in the early 1980's coaxed the European scientific community in the direction of a centrally organised transnational research programme. The success of this initiative is best measured by reference to the 1987 BAP statistics: a total of 262 research contracts in the entire programme of which 73 related to Enzyme Engineering were presented at the conference by their project leaders and numerous coworkers from all member states of the community.

BAP represents the second phase of the initiative and has obviously learned lessons from its predecessor. One of these lessons is that is is not enough to support 'applied enzymology' without simultaneously nurturing the supporting technologies. The present programme comprises many of those subject areas present in BEP, such as enzyme and protein engineering, genetic engineering, cell culture technology and culture collection. In addition, a subprogramme, 'contextual measures', was convened jointly, bringing to the discussion such diverse topics as data capture techniques, data banks, computer models and advanced software. The combination of these basic scientific disciplines with the technological 'tools' will, it is hoped, place European industry and agriculture in a position to more readily exploit the discoveries of biotechnology into the $1990 \mathrm{~s}$.

The meeting began with an opening address by Alan Fersht. On this occasion Fersht talked about his highly original work on subtilisin in which subtle changes in the position and character of charged groups at the periphery of an active site can lead to dramatic effects on catalytic activities at different $\mathrm{pHs}$. These activity changes are due to through-water electrostatic effects on the pKa of the active site histidine and in the most dramatic case 